\title{
Representation of Functions by Walsh's Series with Monotone Coefficients
}

\author{
Razmik Melikbekyan ${ }^{1}$
}

${ }^{1}$ Yerevan State University, Yerevan, Armenia

Correspondence: Razmik Melikbekyan, Department of Physics, Yerevan State University, Alex Manukian 1, Yerevan 0025, Armenia. E-mail: melikbekyan@yahoo.com

Received: December 13, 2012 Accepted: January 21, 2013 Online Published: February 19, 2013

doi:10.5539/jmr.v5n1p107 URL: http://dx.doi.org/10.5539/jmr.v5n1p107

\section{Abstract}

There exists a series in the Walsh system $\left\{\varphi_{n}\right\}$ of the form

$$
\sum_{i=1}^{\infty} a_{i} \varphi_{i}, \quad \text { with } \quad\left|a_{i}\right| \searrow 0,
$$

that possess the following properties:

For any $\epsilon>0$ and any function $f \in L^{1}(0,1)$ there exists set $E \subset[0,1](|E|>1-\epsilon)$ and a sequence $\left\{\delta_{i}\right\}_{i=0}^{\infty}$, $\delta_{i}=0$ or 1 , such that the series

$$
\sum_{i=0}^{\infty} \delta_{i} a_{i} \varphi_{i}
$$

converges to $f$ on $E$ in the $L^{1}(0,1)$-metric and on $[0,1] \backslash E$ in the $L^{r}([0,1] \backslash E)$ metric for all $r \in(0,1)$.

Keywords: orthonormal system, convergence, functional series

AMS 2000 Mathematics Subject Classification: 42C10,42C20

\section{Introduction}

The problem of representing a function $f$ by a series in classical and general orthonormal systems has a long history.

A question posed by Lusin in 1915 asks whether it be possible to find for every measurable function $[0,2 \pi]$ a trigonometric series, with coefficient sequence converging to function. For real-valued functions, this question was given an affirmative answer by Men'shov in 1941.

There are many other works (see Talalian, 1960; Men'shov, 1947, 1941; Grigorian, 1999, 2003, 2000; Ul'janov, 1972; Ivanov, 1989; Krotov, 1977; Kozlov, 1950) devoted to representations of functions by series in classical and general orthonormal systems and the existence of different types of universal series in the sense of convergence almost everywhere and by measure.

The papers by Men'shov (1947) and Kozlov (1950) were the first to construct some ordinary universal trigonometric series in the class of all measurable functions in the sense of a.e. convergence.

Grigoryan (2009) proved the following important result:

For any $\epsilon>0$ and any function $f \in L[0,1]$ there exists a sequence $\left\{\delta_{i}\right\}_{i=0}^{\infty}, \delta_{i}=0$ or 1 , such that the series with monotone coefficients

$$
\sum_{i=0}^{\infty} \delta_{i} a_{i} \varphi_{i}
$$

converges to $f$ in the $L^{1}(E)$-metric. In Grigoryan's paper properties of series outside the $E$ remained open. In this paper we succeed to ensure the convergence of the series to $f$ outside $E$ in weaker metric. Note that convergence is impossible in the same metric. 
Let $r$ be the periodic function, of least period 1 , defined on $[0,1)$ by

$$
r=\chi_{[0,1 / 2)}-\chi_{[1 / 2,1)} .
$$

The Rademacher system, $R=r_{n}: n=0,1, \ldots$, is defined by the conditions

$$
r_{n}(x)=r\left(2^{n} x\right), \forall x \in R, n=0,1, \ldots,
$$

and, in the ordering employed by Payley (see Golubov, Efimov, \& Skvartsov, 1987; Paley, 1932), the n-th element of the Walsh system $\left\{\varphi_{n}\right\}$ is given by

$$
\varphi_{n}=\prod_{k=0}^{\infty} r_{k}^{n_{k}}
$$

where $\sum_{k=0}^{\infty} n_{k} 2^{k}$ is the unique binary expansion of $n$, with each $n_{k}$ either 0 or 1 .

In the present work we prove the following theorem:

Theorem There exists a series in the Walsh system of the form

$$
\sum_{i=1}^{\infty} a_{i} \varphi_{i}, \quad \text { with } \quad\left|a_{i}\right| \searrow 0,
$$

that possess the following properties:

For any $\epsilon>0$ and any function $f \in L^{1}(0,1)$ there exists set $E \subset[0,1](|E|>1-\epsilon)$ and a sequence $\left\{\delta_{i}\right\}_{i=0}^{\infty}, \delta_{i}=$ 0 or 1 , such that the series

$$
\sum_{i=0}^{\infty} \delta_{i} a_{i} \varphi_{i}
$$

converges to $f$ on $E$ in the $L^{1}(0,1)$-metric and on $[0,1] \backslash E$ in the $L^{r}([0,1] \backslash E)$ metric for all $r \in(0,1)$.

The following problem remains open: is the Theorem true for the trigonometric system?

\section{Basic Concepts and Terminology}

We put

$$
I_{k}^{(j)}(x)=\left\{\begin{array}{ll}
1, & \text { if } x \in[0,1] \backslash \Delta_{k}^{(j)}, \\
1-2^{k}, & \text { if } x \in \Delta_{k}^{(j)}=\left(\frac{j-1}{2^{k}}, \frac{j}{2^{k}}\right),
\end{array} k=1,2, \ldots, 1 \leq j \leq 2^{k},\right.
$$

and periodically extend these functions on $R^{1}$ with period 1 .

By $\chi_{E}(x)$ we denote the characteristic function of the set $E$, i.e.

$$
\chi_{E}(x)= \begin{cases}1, & \text { if } x \in E, \\ 0, & \text { if } x \notin E .\end{cases}
$$

Then, clearly

$$
I_{k}^{(j)}(x)=\varphi_{0}(x)-2^{k} \cdot \chi_{\Delta_{k}^{(j)}}(x),
$$

and let for the natural numbers $k \geq 1$, and $j \in\left[1,2^{k}\right]$

$$
\begin{gathered}
b_{i}\left(\chi_{\Delta_{k}^{(j)}}\right)=\int_{0}^{1} \chi_{\Delta_{k}^{(j)}}(x) \varphi_{i}(x) d x= \pm \frac{1}{2^{k}}, 0 \leq i<2^{k} \\
a_{i}\left(I_{k}^{(j)}\right)=\int_{0}^{1} I_{k}^{(j)}(x) \varphi_{i}(x) d x= \begin{cases}0, & \text { if } i=0, \text { if } i \geq 2^{k}, \\
\pm 1, & \text { if } 1 \leq i<2^{k} .\end{cases}
\end{gathered}
$$

Hence

$$
\chi_{\Delta_{k}^{(j)}}(x)=\sum_{i=0}^{2^{k}-1} b_{i}\left(\chi_{\Delta_{k}^{(j)}}\right) \varphi_{i}(x)
$$




$$
I_{k}^{(j)}(x)=\sum_{i=1}^{2^{k}-1} a_{i}\left(I_{k}^{(j)}\right) \varphi_{i}(x)
$$

Lemma 1 Let dyadic interval $\Delta=\Delta_{m}^{(k)}=\left((k-1) / 2^{m} ; k / 2^{m}\right), \quad k \in\left[1,2^{m}\right]$ and numbers $N_{0} \in N, \gamma \neq 0, \epsilon \in$ $(0,1), r_{2} \in(0,1)$ be given. Then there exists a measurable set $E \subset[0,1]$ and a polynomial $Q$ in the Walsh system $\left\{\varphi_{k}\right\}$ of the following form

$$
Q=\sum_{k=N_{0}}^{N} a_{k} \varphi_{k}
$$

which satisfy the following conditions:

1) the coefficients $\left\{a_{k}\right\}_{k=N_{0}}^{N}$ are 0 or $\pm \gamma|\Delta|$,

2) $|E|>(1-\varepsilon)|\Delta|$,

3) $Q(x)=\left\{\begin{array}{ll}\gamma: & \text { if } x \in E \\ 0: & \text { if } x \notin \Delta\end{array}\right.$,

4) $\int_{\Delta}\left|\gamma \chi_{\Delta}(x)-Q(x)\right|^{r} d x<\epsilon|\Delta \| \gamma|^{r}, \forall r \in\left(0, r_{2}\right)$,

5) $\max _{N_{0} \leq m \leq N} \int_{0}^{1}\left|\sum_{k=N_{0}}^{m} a_{k} \varphi_{k}(x)\right| d x<\frac{|\gamma \| \Delta|^{\frac{1}{2}}}{\epsilon^{\frac{1}{\left(1-r_{2}\right)}}}$.

Proof. Let

$$
v_{0}=\left[\frac{1}{1-r_{2}} \log _{2} \frac{1}{\epsilon}\right] ; s=\left[\log _{2} N_{0}\right]+m .
$$

We define the polynomial $Q(x)$ and the numbers $c_{n}, a_{i}$ and $b_{j}$ in the following form:

$$
\begin{gathered}
Q(x)=\gamma \cdot \chi_{\Delta_{m}^{(k)}}(x) \cdot I_{\nu_{0}}^{(1)}\left(2^{s} x\right), x \in[0 ; 1] . \\
c_{n}=c_{n}(Q)=\int_{0}^{1} Q(x) \varphi_{n}(x) d x, \forall n \geq 0, \\
b_{i}=b_{i}\left(\chi_{\Delta_{m}^{(k)}}\right), 0 \leq i<2^{m}, a_{j}=a_{j}\left(I_{\nu_{0}}^{(1)}\right), 0<j<2^{\nu_{0}} .
\end{gathered}
$$

Taking into consideration the following equation

$$
\varphi_{i}(x) \cdot \varphi_{j}\left(2^{s} x\right)=\varphi_{j \cdot 2^{s}+i}(x), \text { if } 0 \leq i, j<2^{s} \quad(\text { see }(1)),
$$

and having the following relations (5)-(8) and (10)-(12), we obtain that the polynomial $Q(x)$ has the following form:

$$
Q(x)=\gamma \cdot \sum_{i=0}^{2^{m}-1} b_{i} \varphi_{i}(x) \cdot \sum_{j=1}^{2^{v_{0}-1}} a_{j} \varphi_{j}\left(2^{s} x\right)=\gamma \cdot \sum_{j=1}^{2^{v_{0}-1}} a_{j} \cdot \sum_{i=0}^{2^{m}-1} b_{i} \varphi_{j \cdot 2^{s}+i}(x)=\sum_{k=N_{0}}^{\bar{N}} c_{k} \varphi_{k}(x),
$$

where

$$
c_{k}=c_{k}(Q)=\left\{\begin{array}{ll} 
\pm \frac{\gamma}{2^{m}} \text { or } 0, & \text { if } k \in\left[N_{0}, \bar{N}\right], \\
0, & \text { if } k \notin\left[N_{0}, \bar{N}\right],
\end{array} \quad \bar{N}=2^{s+\nu_{0}}+2^{m}-2^{s}-1 .\right.
$$

Then let

$$
E=\{x ; Q(x)=\gamma\}
$$

Clearly that (see (2) and (10)),

$$
\begin{gathered}
|E|=2^{-m}\left(1-2^{-\nu_{0}}\right)>(1-\epsilon)|\Delta|, \\
Q(x)= \begin{cases}\gamma, & \text { if } x \in E, \\
\gamma\left(1-2^{\nu_{0}}\right), & \text { if } x \in \Delta \backslash E, \\
0, & \text { if } x \notin \Delta .\end{cases}
\end{gathered}
$$

Hence and from (9) for all $r \in\left(0, r_{2}\right)$ we obtain

$$
\int_{\Delta}\left|\gamma \chi_{1}(x)-Q(x)\right|^{r} d x=\int_{\Delta \backslash E}\left|2^{\nu_{0}} \gamma\right|^{r} d x \leq|\gamma|^{r}|\Delta|\left(\frac{1}{2^{\nu_{0}}}\right)^{\left(1-r_{2}\right)}<|\gamma|^{r}|\Delta| \epsilon .
$$


By Bessel's inequality and by (13)-(16) we have

$$
\max _{N_{0} \leq m \leq N} \int_{0}^{1}\left|\sum_{k=N_{0}}^{m} a_{k} \varphi_{k}(x)\right| d x \leq \max _{N_{0} \leq m \leq N}\left(\int_{0}^{1}\left|\sum_{k=N_{0}}^{m} a_{k} \varphi_{k}(x)\right|^{2} d x\right)^{\frac{1}{2}} \leq\left(\int_{0}^{1} Q^{2}(x) d x\right)^{\frac{1}{2}} \leq \leq 2^{\nu_{0}}|\gamma||\Delta|^{\frac{1}{2}} \leq \frac{2|\gamma||\Delta|^{\frac{1}{2}}}{\epsilon^{\frac{1}{\left(1-r_{2}\right)}}}
$$

Lemma 1 is proved.

Lemma 2 Let given the numbers $\tilde{N} \in N, 0<\epsilon<1,0<r_{1}<r_{2}<1$. Then for any function $f \in L^{1}(0,1),\|f\|_{L_{1}}>0$, one can find a set $E \subset[0,1]$ and a polynomial in the Walsh system

$$
Q=\sum_{k=\tilde{N}+1}^{M} a_{k} \varphi_{k}
$$

satisfying the following conditions:

1) $0 \leq a_{k}<\epsilon$ and the non-zero coefficients in $\left\{a_{k}\right\}_{k=\tilde{N}+1}^{M}$ are in decreasing order,

2) $|E|>1-\epsilon$,

3) $\int_{0}^{1}|Q(x)-f(x)|^{r} d x<\epsilon, \forall r \in\left(r_{1}, r_{2}\right)$,

4) $\int_{E}|Q(x)-f(x)| d x<\epsilon$,

5) $\max _{\tilde{N}+1 \leq m \leq M} \int_{0}^{1}\left|\sum_{k=\tilde{N}+1}^{m} a_{k} \varphi_{k}(x)\right|^{r} d x<\int_{0}^{1}|f|^{r} d x+\epsilon, \forall r \in\left(r_{1}, r_{2}\right)$,

6) $\max _{\tilde{N}+1 \leq m \leq M}\left|\int_{E} \sum_{k=\tilde{N}+1}^{m} a_{k} \varphi_{k}(x) d x\right|<\int_{E}|f(x)| d x+\epsilon$.

Proof. Let

$$
\delta=\frac{\epsilon}{2} \min \left\{\left(\frac{\epsilon}{2}\right)^{\frac{1-r_{1}}{r_{1}}} ;\left(\sup _{r \in\left(r_{1}, r_{2}\right)} \int_{0}^{1}|f(x)|^{r} d x+1\right)^{-1}\right\} .
$$

We choose some non-overlapping binary intervals $\left\{\Delta_{v}\right\}_{v=1}^{\nu_{0}}$ and a step function

$$
\varphi(x)=\sum_{v=1}^{\nu_{0}} \gamma_{\nu} \cdot \chi_{\triangle_{\nu}}(x)
$$

satisfying the conditions

$$
\begin{gathered}
\sum_{v=1}^{\nu_{0}}\left|\Delta_{v}\right|=1, \max _{1 \leq v \leq \nu_{0}}\left|\gamma_{v}\right|\left|\Delta_{v}\right|^{\frac{1}{2}}<\frac{\epsilon}{4} \cdot \delta^{\frac{1}{1-r_{2}}}, \\
\frac{\epsilon}{2}>\left|\gamma_{1}\right|\left|\Delta_{1}\right|>\ldots>\left|\gamma_{v}\right|\left|\Delta_{v}\right|>\ldots>\left|\gamma_{\nu_{0}}\right|\left|\Delta_{v_{0}}\right|>0, \\
\left(\int_{0}^{1}|f-\varphi| d x\right)<\delta^{2} .
\end{gathered}
$$

Now let

$$
B=\{x \in[0,1]:|f(x)-\varphi(x)|<\delta\} .
$$

Then by (17), (21) and (22)

$$
|B|>1-\frac{\epsilon}{2} .
$$

Successively applying Lemma 1 , we determine some sets $E_{v} \subset[0,1]$ and polynomials

$$
Q_{v}=\sum_{j=m_{v-1}}^{m_{v}-1} a_{j} \varphi_{j},\left(m_{0}=\bar{N}+1\right), v=1, \ldots, v_{0},
$$


where $a_{j}=0$ or $\pm \gamma_{j}\left|\Delta_{j}\right|$, if $j \in\left[m_{v-1}, m_{v}\right)$,

$$
\begin{gathered}
\left|E_{v}\right|>\left(1-\frac{\epsilon}{2}\right) \cdot\left|\Delta_{v}\right|, \\
Q_{v}= \begin{cases}\gamma_{v}: & \text { if } x \in E_{v} \\
0: & \text { if } x \notin \Delta_{v}\end{cases} \\
\int_{\Delta_{v}}\left|\gamma_{v} \chi_{\Delta_{v}}(x)-Q_{v}(x)\right|^{r} d x<\delta\left|\Delta_{v}\right|\left|\gamma_{v}\right|^{r}, \forall r \in\left(0, r_{2}\right), \\
\max _{m_{v-1} \leq m \leq m_{v}-1}\left(\int_{0}^{1}\left|\sum_{k=m_{v-1}}^{m} a_{k} \varphi_{k}(x)\right| d x\right)<\frac{2\left|\gamma_{v}\right|\left|\Delta_{v}\right|^{\frac{1}{2}}}{\delta^{\frac{1}{\left.1-r_{2}\right)}}},
\end{gathered}
$$

Then let

$$
\begin{gathered}
E=\bigcup_{v=1}^{\nu_{0}} E_{\nu} \cap B, \\
Q=\sum_{\nu=1}^{\nu_{0}} Q_{\nu}=\sum_{k=\tilde{N}+1}^{M} a_{k} \varphi_{k},
\end{gathered}
$$

From (20),(23), (24) (25) and (29) follows, that

$$
|E|>1-\epsilon .
$$

and $0 \leq a_{k}<\epsilon$ and the non-zero coefficients in $\left\{a_{k}\right\}_{k=\tilde{N}+1}^{M}$ are in decreasing order.

By (17),(18),(21),(30) for all $r \in\left(r_{1}, r_{2}\right)$ we have

$$
\int_{0}^{1}|Q(x)-f(x)|^{r} d x \leq\left(\int_{0}^{1}|f(x)-\varphi(x)| d x\right)^{r}+\sum_{\nu=1}^{\nu_{0}} \int_{0}^{1}\left|\gamma_{\nu} \chi_{\Delta_{\nu}}(x)-Q_{\nu}(x)\right|^{r} d x<\delta^{r_{1}}+\delta \cdot \int_{0}^{1}|\varphi(x)|^{r}<\epsilon,
$$

i.e. the statements 1), 2), 3) of Lemma 2 are valid.

To verify the statements 5) and 6), for any $\tilde{N}<m \leq M$ determine $v$ from the condition $m_{v-1} \leq m<m_{v}$. Then by (23) and (30)

$$
\sum_{k=\tilde{N}+1}^{m} a_{k} \varphi_{k}=\sum_{n=1}^{v-1} Q_{n}+\sum_{k=m_{v}-1}^{m} a_{k} \varphi_{k}
$$

Hence by (17)-(19), (21), (26), (27) and (28) we obtain that for all $r \in\left(r_{1}, r_{2}\right)$

$$
\begin{gathered}
\int_{0}^{1}\left|\sum_{k=\tilde{N}+1}^{m} a_{k} \varphi_{k}(x)\right|^{r} d x \leq \sum_{n=1}^{v-1} \int_{0}^{1}\left|Q_{n}(x)-\gamma_{n} \chi_{\Delta_{n}}(x)\right|^{r} d x+\sum_{n=1}^{v_{0}} \int_{0}^{1}\left|\gamma_{n} \chi_{\Delta_{n}}(x)\right|^{r} d x+ \\
+\int_{0}^{1}\left|\sum_{k=m_{\nu}-1}^{m} a_{k} \varphi_{k}(x)\right|^{r} \leq \delta \cdot \int_{0}^{1}|\varphi(x)|^{r} d x+\int_{0}^{1}|f(x)|^{r} d x+\delta^{r_{1}}+\frac{\epsilon}{2} \leq \int_{0}^{1}|f(x)|^{r} d x+\epsilon .
\end{gathered}
$$

Since for any point $x \in E, Q(x)=\varphi(x)$ (see (26),(29) and (30)), then from the conditions (17),(21), (28), (29) and (31), we have

$$
\begin{gathered}
\int_{E}|Q(x)-f(x)| d x=\int_{E}|\varphi(x)-f(x)| d x<\epsilon . \\
\left|\int_{E}\right| \sum_{k=\tilde{N}+1}^{m} a_{k} \varphi_{k}(x) d x\left|\leq \int_{E}\right| \sum_{n=1}^{v-1} \gamma_{n} \chi_{\Delta_{n}}(x) d x\left|+\int_{E}\right| \sum_{n=m_{v}-1}^{m} a_{n} \varphi_{n}(x) d x\left|d x \leq \int_{E}\right| \varphi(x)\left|d x+\frac{\epsilon}{2} \leq \int_{E}\right| f(x) \mid d x+\epsilon .
\end{gathered}
$$

Lemma 2 is proved.

\section{Proof of the Theorem}

Let

$$
\left\{f_{k}(x)\right\}_{k=1}^{\infty}, \quad x \in[0,1]
$$


be the sequence of all algebraic polynomials with rational coefficients. Applying repeatedly Lemma 2, we obtain sequences of $\left\{E_{k}\right\}_{k=1}^{\infty}$ sets and polynomials in the Walsh systems $\left\{\varphi_{n}(x)\right\}$

$$
Q_{k}(x)=\sum_{i=N_{k}}^{M_{k}} a_{n_{i}} \varphi_{n_{i}}(x),
$$

where

$$
N_{1}=1 ; N_{k}=M_{k-1}+1, k \geq 2,
$$

which satisfy the following conditions:

$$
\begin{gathered}
2^{-j}>\left|a_{n_{i}}\right| \geq\left|a_{n_{i+1}}\right|>0, \forall i \in\left[N_{k}, M_{k}\right], k=1,2, \ldots, \\
\int_{0}^{1}\left|Q_{k}(x)-f_{k}(x)\right|^{r} d x<2^{-4(k+1)}, \forall r \in\left[2^{-k}, 1-2^{-k}\right], \\
\int_{E_{k}}\left|Q_{k}(x)-f_{k}(x)\right| d x<2^{-4 k}, \\
\max _{N_{k} \leq m \leq M_{k}} \int_{0}^{1}\left|\sum_{i=N_{k}}^{m} a_{n_{i}} \varphi_{n_{i}}(x)\right|^{r} d x<\int_{0}^{1}\left|f_{j}(x)\right|^{r} d x+2^{-k-1}, k=1,2, \ldots, \\
\max _{N_{k} \leq m \leq M_{k}} \int_{E_{k}}\left|\sum_{i=N_{k}}^{m} a_{n_{i}} \varphi_{n_{i}}(x)\right| d x<\int_{0}^{1}\left|f_{k}(x)\right| d x+2^{-k-1}, \\
\left|E_{k}\right|>1-2^{-k-1} .
\end{gathered}
$$

Consider a series

$$
\sum_{s=1}^{\infty} a_{s} \varphi_{s}(x), \text { where } a_{s}=a_{n_{i}} \text { if } s \in\left[n_{i}, n_{i+1}\right) \quad \text { (see (33)), }
$$

and a set

$$
E=\bigcap_{k=k_{0}}^{\infty} E_{k}, \text { where } k_{0}=\left[\log _{2} \frac{1}{\epsilon}\right]+1 .
$$

Clearly that (see (33), (34) and (39)-(41))

$$
\left|a_{k}\right| \searrow 0 \quad \text { and } \quad|E|>1-\epsilon .
$$

Let $r \in(0,1)$, then for some $j_{0}>1$ we have $r \in\left(\frac{1}{j_{0}}, 1-\frac{1}{j_{0}}\right)$, and let $f(x) \in L_{(0,1)}^{r}$.

We choose some $f_{v_{1}}(x), v_{1}>j_{0}$, from sequence (32), to have

$$
\int_{0}^{1}\left|f(x)-f_{v_{1}}(x)\right|^{r} d x<2^{-4}
$$

Denote that the numbers $j_{0}<v_{1}<\ldots<v_{q-1}$ and polynomials $Q_{v_{1}}(x), \ldots, Q_{v_{q-1}}(x)$ are already determined satisfying to the following conditions:

$$
\begin{gathered}
\int_{0}^{1}\left|f(x)-\sum_{n=1}^{s} Q_{v_{n}}(x)\right|^{r} d x<2^{-4 s}, s \in[2, q-1], \\
\max _{N_{v_{n}} \leq m \leq M_{v_{n}}} \int_{0}^{1}\left|\sum_{i=N_{v_{n}}}^{m} a_{n_{i}} \varphi_{n_{i}}(x)\right|^{r} d x<2^{-n}, n \in[2, q-1]
\end{gathered}
$$

Let a function $f_{v_{q}}(x), v_{q}>v_{q-1}$ is from the sequence (32) such that

$$
\int_{0}^{1}\left|\left[f(x)-\sum_{j=1}^{q-1} Q_{j}^{\left(v_{j}\right)}(x)\right]-f_{v_{q}}(x)\right|^{r} d x<2^{-4(q+1)} .
$$


Hence by (42) we obtain

$$
\int_{0}^{1}\left|f_{v_{q}}\right|^{r} d x<2^{-q-1} .
$$

From the conditions (35), (37), (44) and (45) follows that

$$
\begin{gathered}
\int_{0}^{1}\left|f(x)-\sum_{j=1}^{q} Q_{v_{j}}(x)\right|^{r} d x<2^{-4 q}, \\
\max _{N_{v_{q}} \leq m \leq M_{v_{q}}} \int_{0}^{1}\left|\sum_{i=N_{v_{q}}}^{m} a_{n_{i}} \varphi_{n_{i}}(x)\right|^{r} d x<2^{-q},
\end{gathered}
$$

Then we obtain that the series

$$
\sum_{k=1}^{\infty} \delta_{k} a_{k} \varphi_{k}(x) \quad(\text { see }(40))
$$

where

$$
\delta_{k}= \begin{cases}1, & \text { if } k=n_{i}, \text { where } i=\bigcup_{q=1}^{\infty}\left[N_{v_{q}}, M_{v_{q}}\right] \\ 0, & \text { otherwise }\end{cases}
$$

converges to $f(x)$ in the $L_{(0,1)}^{r}$.

From the conditions (36), (38), (44) and (41) follows that

$$
\sum_{k=1}^{\infty} \delta_{k} a_{k} \varphi_{k}(x) \quad(\text { see }(40))
$$

series converges to $f(x)$ in the $L^{1}(E)$ metric.

Theorem is proved.

\section{References}

Golubov, B. I., Efimov, A. F., \& Skvartsov, V. A. (1987). Series and transformations of Walsh. Moskow.

Grigorian, M. G. (1999). On the representation of functions by orthogonal series in weighted $L^{p}$ spaces. Studia. Math., 134(3), 207-216.

Grigorian, M. G. (2000). Universal or quasiuniversal in $L_{[0,1]}^{p}$ orthogonal series. Journal of Contemporary Mathematical Analysis, 35(4), 44-64.

Grigorian, M. G. (2003). On the $L_{\mu}^{p}$-strong property of orthonormal systems. Matem. Sbornik, 194(10), $1503-1532$.

Grigorian, M. G. (2009). Series byWalsh's System with Monotone Coefficients. International Journal of Modern Mathematics, 4(3), 259-267.

Ivanov, V. I. (1989). Representation of functions by series in symmetric metric spaces without linear functionals [in Russian]. Trudy MIAN SSSR, 189, 34-77.

Kozlov, V. Y. (1950). On the complete systems of orthogonal functions [in Russian]. Mat. Sbornik, 26(3), $351-364$.

Krotov, V. G. (1977). Representation of measurable functions by series with respect to Faber-Schauder system and universal series. Izv. AN. SSSR, Ser. http://dx.doi.org/10.1070/IM1977v011n01ABEH001706

Men'shov, D. E. (1941). Sur la representation des fonctions measurables des series trigonometriques. Mat. Sbornik, 9, 667-692.

Men'shov, D. E. (1947). On the partial sums of trigonometric series [in Russian]. Mat. Sbornik, 20(2), $197-238$.

Paley, R. E. A. C. (1932). A remarkable set of orthogonal functions. Proc. London Math. Soc., 34, 241-279.

Talalian, A. A. (1960). Representation of measurable functions by series [in Russian]. UMN, 15(5), 567-604. http://dx.doi.org/10.1070/RM1960v015n05ABEH001115

Ul'janov, P. L. (1972). Representation of functions by series and classes $\varphi(L)$ [in Russian]. UMN, 25(2), 3-52. 